\title{
Desmitificación de tres suposiciones en torno a la ética y su relevancia en la formación de profesionales
}

\author{
Demystification of three assumptions about the ethics and revelance in the professional training
}

\author{
Eduardo F. Ortiz C. ${ }^{1}$
}

\begin{abstract}
Resumen
Con el objetico de desmitificar tres suposiciones en torno a la ética y su relevancia en la formación de profesionales, el autor examina tres suposiciones en torno a la ética, las cuales se expresan en frases comunes que las personas repiten en el ámbito de la universidad, específicamente en una de carreras científico-técnicas como la UNALM. La primera suposición define a la ciencia como autómata y autosuficiente, es decir, como si tuviera independencia de la ética, lo cual provoca la instrumentalización de personas y del medio ambiente; este divorcio debe resolverse responsabilizándose con la formación de profesionales que encarnen una racionalidad moral capaz de orientar de manera humanística la acción de la ciencia en los entornos donde opera. Una segunda suposición se refiere a la ética como recipiente de principios que no cambian desde su adquisición en la infancia, de donde deducen su irrelevancia en la formación profesional; en ese sentido, el autor muestra que, el ethos o principios inconscientes adquiridos en los horizontes tempranos de la biografía y la cultura, no tienen todo el poder de determinar la acción del sujeto cuando a este se le forma como un sujeto consciente, crítico y autónomo. Finalmente, la tercera suposición refleja una concepción individualista de la ética, asumiendo que esta consiste en un conjunto de decisiones dirigidas a la autorrealización individual que está desvinculada de los intereses políticos de la comunidad; ante ello, esta investigación describe la formación humanística como una contribución para la creación de espacios y redes sociales que sostengan el compromiso participativo y el consenso de objetivos y proyectos comunes.
\end{abstract}

Palabras Clave: Ciencia moderna empírica, determinismo inconsciente, ética formación humanística, individualismo instrumentalización.

\begin{abstract}
The author examines three assumptions about ethics, which are expressed in common phrases that people repeat in the field of college, specifically in scientific and technical careers as taught in UNALM. The first assumption defines science as automata and self-sufficient, ie, as if independence of ethics, which causes the exploitation of people and the environment; this divorce must be solved taking responsibility with the training of professionals who embody a moral rationality capable of guiding action humanistic way of science in environments where it operates. A second assumption relates to ethics as container unchanging principles since its acquisition in childhood, from which derive their irrelevance in professional training; in this sense, the author shows that the ethos or unconscious principles acquired in the early horizons biography and culture, not have all the power to determine the action of the subject when this can be formed as a conscious subject, critical and autonomous. Finally, the third assumption reflects an individualistic conception of ethics, assuming that this is a set of decisions for individual self-realization that is unrelated to the political interests of the community; to do so, this research describes the humanistic education as a contribution to the creation of spaces and social networks that sustain the participatory engagement and consensus of common objectives and projects.
\end{abstract}

Keywords: Ethics, individualism instrumentation, modern empirical science, training humanistic, unconscious determinism

\section{Introducción}

Al verificar en el ámbito académico, la tendencia de querer enseñar a los demás por encima de facilitar un diálogo con ellos, esta investigación se inscribe en el esfuerzo moral de cultivar la escucha respetuosa de aquello que la gente tiene por cierto. Por ello, antes de proceder al análisis sistemático, se ha resuelto determinar algunas suposiciones con respecto a la ética, las cuales representan un complejo fenómeno que no queremos marginar sino comprender en sus raíces ontológicas. Este fenómeno podría describirse de manera tripartita. Primero: la ciencia empírica moderna y sus tecnologías actúan en la modernidad sin límites morales que la orienten. Segundo: los presupuestos morales del inconsciente biográfico y cultural, determinan el comportamiento humano cuando no se los analiza sistemáticamente. Tercero: los asuntos morales no son sólo individuales y por tanto no pueden resolverse de manera privada y sin compromisos 
colectivos o políticos.

El problema del presente estudio es que estos supuestos sobre la ética carecen de fundamentación, lo cual podría conducir a ciertas prácticas inmorales cuya imprecisión es necesario señalar. En este sentido, se ha procedido a la discusión de ciertas frases detectadas como más comunes y características que, en el contexto de la universidad, encarnan tales supuestos: La primera: “...Está claro: la ética es una cosa y la ciencia es otra ... "; la segunda: "Yo no entiendo... qué necesidad tenemos de enseñar ética si la ética se aprende en la casa..."; y la tercera: "Bueno pues, finalmente la ética ya depende de cada uno...". Dichas de esa forma parecerían, hasta un punto ciertas e incluso inocuas, pero si exploramos sus consecuencias podríamos llegar a la conclusión de no estar totalmente de acuerdo con ellas.

Dada la complejidad del fenómeno, no vamos a reducir el problema, afirmando simplemente que son falsas o que son ligeras y apresuradas, porque en sentido positivo, representan una manera intuitiva de expresar supuestos que necesitan de esclarecimiento o conceptualización y al mismo tiempo un reclamo de sentido que no está satisfecho y requiere articulación. Por eso, el enfoque metodológico de la investigación es cualitativo y el alcance o nivel explicativo. El objetivo es presentar el análisis de tres creencias o suposiciones que se tematizan en las tres frases cotidianas que utilizan las personas, con la intención de verificar su veracidad racional y desmitificar el discurso que la acompaña. La meta es poder devolverlas en forma sistematizada a la comunidad y así continuar con el diálogo hacia la construcción de una racionalidad moral más compleja e integradora. Para evaluar estos supuestos se han revisado los conceptos del filósofo alemán Hans-George Gadamer respecto a la ciencia moderna empírica en la era de la civilización tecnológica y las consecuencias de desorientación moral que provocó, así como la propuesta de una hermenéutica antropológica del sujeto moderno acerca de su identidad en este nuevo contexto. Por otro lado, las categorías de ciencia como saber falible y perfectible, la filosofía de la ciencia que impide a la ciencia estrechar su visión unilateral, especializada y aislada de otras perspectivas disciplinarias, la concepción de hombre inconcluso e interminable, propuestas por el filósofo Mario Bunge. Asimismo, los conceptos de individualismo, primacía de la razón instrumental, atomismo moral, propias de la modernidad, horizontes morales ineludibles y carácter dialógico de la vida humana, entre otros, del filósofo canadiense Charles Taylor. También, las categorías del filósofo alemán Jürgen Habermas sobre práctica deliberativa para el entendimiento lingüístico en estructuras comunes de comunicación y de socialización intersubjetiva, competencia cooperativa en la comunidad inclusiva, principalmente. Finalmente, los conceptos de deformación onírica durante los sueños que opera el inconsciente como forma de liberación de sus represiones y que utiliza como objeto transferencial, del neurólogo austriaco y fundador del psicoanálisis Sigmund Freud.

\section{Materiales y métodos}

El "lugar" de ejecución de la presente investigación es la ética en la formación universitaria y la repercusión de algunos de sus supuestos en la enseñanza de las carreras científico-técnicas. La metodología está basada en un enfoque cualitativo, ya que busca comprender la realidad a partir de las interpretaciones que los propios actores del ámbito universitario hacen de ella, esto es, estudiantes, docentes e investigadores. En ese sentido, en el diseño de investigación el alcance o nivel del trabajo se define como explicativo; en primer lugar, porque se llega hasta las causas del fenómeno al mismo tiempo que se lo circunscribe en el escenario de las condiciones en que ocurre; y en segundo lugar, porque utiliza algunas categorías de la filosofía y del psicoanálisis para inferir probabilidades o posibles consecuencias.

\section{Resultados y discusión}

Primera suposición: "Está claro: la ética es una cosa y la ciencia es otra".

Si bien la ciencia y la tecnología modernas, han llevado a la humanidad de una parte, al desarrollo del conocimiento y la creación de instrumentos que le han permitido afrontar las inclemencias de la naturaleza, por otro lado, le han vendido el sueño del progreso ilimitado cuyo resultado terminó siendo devastador en su historia y deshumanizante en su devenir. Pero, ¿cómo es que ellas han provocado en la humanidad este sueño desmedido?

Primero describámoslo para poder interpretarlo. Desde una perspectiva psiconanalítica, podríamos decir que este sueño es como la realización de los deseos que, por diversos motivos, no han podido realizarse durante la vigilia, es decir, en el uso libre de la conciencia. Durante el sueño de "la ciencia ilimitada" se suceden una serie de escenas en las que la tecnología aparece resolviendo los problemas que el ser humano no pretende pensar ni resolver. Él se porta como "paciente" que espera una solución venida de fuera, de una fuerza mayor y opuesta a la fuerza de su yo personal y su conciencia, una solución precisamente tecnológica. En esta misma serie, la ciencia predice desastres naturales y los controla para que no causen ningún estrago; produce alimentos interviniendo sus matrices genéticas con el fin de salvar del hambre al mundo; cura las enfermedades antiguas y las nuevas que van apareciendo.

Esta displicencia de no resolver durante la vida despierta lo que debería el ser humano hacer por él mismo, se manifiesta en la recurrencia inconsciente a la fantasía del sueño. De hecho, Sigmund Freud cuando postula que el sueño es una "deformación onírica" de la realidad, una realización de los deseos reprimidos o una censura inconsciente, precisa que se trata de una "actividad intelectual altamente complicada"(Freud, 1900). En efecto, en el caso que estamos analizando, el sujeto moderno realiza una transferencia, es decir, utiliza en 
su sueño el "disfraz" de la ciencia y transfiere a ella su propia responsabilidad de acción. Para esto, espera a que la conciencia, que es como un centinela, se quede dormida, para empezar a jugar como un niño, liberado de todo esfuerzo y responsabilidad.

Evidentemente, en el fondo sabe que sólo es un sueño, algo imposible, un juego distractor: convertirse en un ser omnipotente cuyos instrumentos eficaces y casi mágicos potencian sus capacidades para resolver el problema con un "touch" (activar algún app o programa especializado de una tablet deslizando el dedo sobre la superficie de la pantalla). Pero al mismo tiempo, en este sueño reprime su conciencia de culpa, de haber sido responsable en la aparición del mismo problema que ahora desea resolver, sólo que sin comprometerse como agente o sujeto "activo" que lo ocasionó. ¿Acaso este sujeto moderno reconoce que con sus nuevos "juguetes", que son la ciencia y su tecnología modernas, ha manipulado la energía nuclear que ocasionó los desastres de gran impacto en el siglo pasado e inicios del presente, o declara que el hambre, ese que supuestamente los transgénicos podrían solucionar, lo ha sido ocasionado él mismo, con las estructuras socioeconómicas injustas que inventó en el pasado próximo o que ciertas enfermedades son producto de la forma de vida que su cultura moderna ha decidido imponer de manera absolutista? A partir de este fenómeno inconsciente puede comprenderse la forma en que la ciencia moderna empírica se construye sobre el supuesto epistemológico de ser un sistema que todo lo puede controlar, predecir y solucionar, con su mecanismo de evasión que es la fantasía de abarcar de manera total el conocimiento de la realidad.

Al respecto, los supuestos contemporáneos de la ciencia, han fundamentado que la investigación científica es progresiva y ayuda al ser humano a alcanzar una reconstrucción conceptual cada vez más amplia, profunda y exacta del mundo, pero al mismo tiempo recuerdan que este es un proceso "falible" y a pesar de ser racional, sistemático y exacto es también "verificable"(Bunge, 2013). Así que esta verificabilidad no puede obviarse como paso de coherencia final para legitimar y regular su conveniencia. Esto parece haber sido olvidado en nuestra época por los que hacen ciencia y los que la procuran o financian.

Aunque parecen neutrales, la ciencia y la tecnología modernas están, en última instancia, en manos de las personas con sus diversos grados de "consistencia ética"2, es decir de conciencia para actuar de maneras

${ }^{2}$ Desde nuestra concepción, consistencia ética se refiere a la formación progresiva de una personalidad moral, es decir, la superación de la vida inconsciente que describe a las personas como sujetos pasivos frente a las leyes imperativas no conceptualizadas que han aprendido de la cultura y de la familia, para llegar a convertirse en sujetos activos que las han tematizado y puesto en evidencia y conciencia. Este proceso, no lo consiguen únicamente por sí mismas, sino en sus relaciones significativas con los otros actores de la comunidad, lo cual les permite convertirse en sujetos más coherentes que pueden decidir lo que razonablemente consideran que es mejor creer. Esto es que, al modificar su sistema de creencias o leyes imperativas aprendidas de manera temprana, se distancian de su determinismo y se emancipan de su dominio, permitiéndoles, dicha liberación, construir un sistema moral más racional y una vida más integral, acorde con la nueva libertad que van conquistando. más o menos racionales, libres y coherentes. En el caso de mayor consistencia, la praxis tecnológica devendrá en el desarrollo de estructuras sociales de justicia y entendimiento $\mathrm{y}$, en el segundo, lo contrario. En efecto, del mismo modo en que el ser humano es perfectible la ciencia igualmente lo es y cuando en esta época comprobamos la falta de distancia o separación ontológica entre ellos, se corre el riesgo de que este ser humano se identifique con la pretendida soberbia de ser imperfectible. Aquí se justifica la necesidad de exigir que, al interior de la ciencia, se procure un espacio de reflexión sistemática sobre la praxis y su contextualización en el horizonte humano y social del entorno: "La investigación tiende a estrechar la visión del científico individual; un único remedio ha resultado eficaz contra la unilateralidad profesional, y es una dosis de filosofía"(Bunge, 2016).

Esto mismo lo formula Hans-George Gadamer en términos hermenéuticos al afirmar que:

"Como es sabido, la moderna ciencia empírica se ha ido liberando, a través de un pesado trabajo crítico, de las ataduras del saber total de la filosofía aristotélica. Para obtener la certeza y la controlabilidad de sus conocimientos y el camino seguro de su progreso, tuvo que pagar el precio de la renuncia a un saber total de este tipo" (Gadamer, 1981).

De hecho, para Platón y Aristóteles, el bien práctico de la vida humana participa del saber de la contemplación del bien, que está por encima de todas las ciencias, las cuales apuntan hacia él. En este sentido original o genético, el bien es el objeto supremo del saber y, el bien práctico que la tecnología pretende, está en relación directa con la ética, proviene y se dirige hacia ella.

En efecto, no puede haber ciencia sin ética, ni tecnología sin un sujeto que la controle. Cuando al sujeto se lo comprende en sentido ético, es decir, en sentido colectivo y relacional, entonces puede llegar a ser consciente de los presupuestos que controlan su acción; por ejemplo, el esclarecimiento de la pulsión de poder podría ser regulada en el contexto de la comunidad que advierte el peligro de interpretarla como mera posesión de poder y la reconduce hacia acciones concretas con miras al bien común. Esta asunción antropológica es fundamental para superar la concepción de la tecnología como un asunto individual y abstracto. Ahora bien, el hecho que la ciencia haya deformado su perfil de ese modo, olvidando que pertenece a una comunidad de científicos que discuten y debaten en torno al progreso, no justifica que conciba al ser humano como sujeto individual y abstraído; no se puede hacer un salto de la epistemología de esta nueva ciencia a una antropología que defina a los científicos de ese mismo modo. La ciencia está en manos de sujetos a quienes no puede dejar de comprenderse en sentido relacional. En consecuencia, es imposible concebir una ciencia sin ética porque la ética no es neutral y debe recaer en nuestra responsabilidad decidir hacia dónde y de qué manera queremos que nos conduzca.

Creer que la ciencia es una cosa que no tiene mucha 
relación con la ética es un engaño peligroso. Si somos honestos con la historia, tendremos que recordar que la ciencia nació como una derivación del quehacer filosófico y se gestó precisamente como el ejercicio de preguntarse con rigurosidad conceptual por los fenómenos observados en la naturaleza, para luego establecer su regularidad mediante la postulación de leyes.

Ciencia sin ética es una consigna que entraña el riesgo de dejar a la ciencia a merced de cualquier tipo de decisiones sin una finalidad que esté orientada más allá de sí misma. Es decir que, al quedar desorientada o alejada de su intención podría desorientarnos o alejarnos de la finalidad última de nuestra condición humana. La ciencia sin ética está desorientada porque va hacia ningún lugar, avanza sin rumbo obedeciendo a los intereses que encuentra a su paso, ni siquiera suyos sino de grupos de poder, como aquellos que hacen funcionar al abusivo sistema neoliberal en economía o al pulpo maléfico del armamentismo o al gran negocio de las farmacéuticas transnacionales. En consecuencia, la ciencia moderna empírica no puede ir dando tumbos; las personas que la controlamos debemos adecuarla a nuestro telos humano o finalidad hacia la que hacemos confluir nuestras acciones y decisiones; ese telos que Aristóteles describía en vinculación con la phronesis o racionalidad capaz de discernir lo que conviene moralmente para procurar el bien, en cada situación de nuestra convivencia en la polis o comunidad ética, con miras a alcanzar finalmente una vida virtuosa y feliz de manera intersubjetiva.

Si en el siglo XIX se propusieron tantas concepciones de mundo dirigidas hacia una interpretación unitaria, es porque la ciencia estaba fracasando en su intento por ofrecer un telos u una orientación integradora que evidentemente el siglo XX tampoco alcanzó ${ }^{3}$. Por ello, la metafísica que se creyó superada, en el sentido que formulaba las preguntas ulteriores acerca del ser en sí, es la que ahora nuevamente orienta el sentido de nuestro ser práctico en el mundo, porque "no es el mundo objetivo de la investigación sino el saber del hombre con respecto a sí mismo y al mundo de sus creaciones en el cual se ha depositado el saber" (Gademer, 1981), lo que representa el todo de orientación del que actualmente carece nuestra cultura y lo que anhela el sujeto moderno para encontrar el sentido otrora perdido. El sujeto moderno sin embargo, corre el riesgo de caminar en círculos concéntricos en vez de caminar hacia adelante, hacia el descubrimiento de horizontes que proceden de otras perspectivas que muestran nuevas posibilidades de sentido antes no conocidas. Es así que Hans-George Gadamer propone que la filosofía de la ciencia tiene que ser hermenéutica del sentido, para que ayude a integrar lo que el sujeto había disociado, ayude a "vincular unitariamente la ciencia y el saber del hombre con respecto a sí mismo a fin de lograr una nueva autocomprensión de la humanidad consigo

${ }^{3}$ En el siglo XIX surgen las grandes filosofías sistémicas que compiten con la ciencia positiva en el contexto de las revoluciones sociales en Europa El racionalismo de Hegel, el positivismo de Comte, el marxismo, el nihilismo de Nietszche, etc. Consultar: COPLESTÓN, Frederick Charles. 2000-2004 Historia de la filosofía. Barcelona: Editorial Ariel. 7, 8 y 9. misma. ${ }^{4}$

Si no configuramos los principios de la ciencia moderna empírica -así como su quehacer tecnológico-, conforme a esta finalidad humana, entonces terminará por deshumanizarnos. Porque lo que aquí se plantea está referido a la ciencia que se apellida moderna y empírica y tecnológica y no simplemente ciencia y tecnología a secas. ¿Acaso el desastre nuclear de Chernobyl, en los años ochenta, no es una prueba fehaciente de la arrogancia de la ciencia autoproclamada como infalible? O si pensamos en las devastaciones más recientes como Tokaimura o el horror en Fukushima, en el 2011, ¿no tendríamos que definir a la tecnología como la causante de los grandes desastres nucleares de los tiempos modernos y de pérdidas humanas y medioambientales tan lamentables como irreparables? Qué diferente en cambio cuando la ciencia y su quehacer tecnológico tienen una orientación ética que va más allá de ellas mismas y sus procedimientos, como sus logros en la comunicación satelital, la descripción de la estructura química del ADN o recientemente, las posibilidades de cura del Alzheimer, entre otros. Ciencia sin ética no se puede concebir, debido al potencial deshumanizador de esta ecuación moral invertida. Ciencia con ética siempre, para que el despliegue de la creatividad en el contexto del rigor metódico de la ciencia impulse la superación de los problemas sociales y el desarrollo en todos sus niveles.

Finalmente, hay preguntas que la ciencia no puede responder, preguntas que están relacionadas con la búsqueda de sentido del ser humano y su quehacer en el mundo, cuestiones afrontadas por las ciencias del espíritu, las ciencias humanas referidas a las expresiones del espíritu humano como el arte, las religiones, las mitologías culturales, las costumbres ancestrales, las formas de convivencia e intercambio, etc. Este reclamo no resuelto, que tampoco se garantiza agotar, pero que es indispensable atender, es tarea del quehacer filosófico y concretamente de la reflexión ética que no se puede eludir en la formación de profesionales, en el contexto de la universidad.

\section{Segunda suposición: "Yo no entiendo... qué necesidad tenemos de enseñar ética si la ética se aprende en la casa".}

Que aprendemos la ética en casa es cierto por un lado y no tan cierto por otro, porque ella es el producto de un largo proceso que va desde la adquisición de elementos inconscientes en la infancia, hasta la construcción consciente de la ética lograda en el intercambio dialógico con los diversos actores de la comunidad.

Lo que en realidad aprendemos en casa es algo a lo que llamaremos ethos ${ }^{5}$, o conjunto de hábitos, costumbres, creencias y normas que por su misma procedencia ${ }^{4}$ Ídem, p. 92.

${ }^{5}$ La sucesión de los conceptos ethos, código valorativo, punto de vista moral y Point Of Moral View, a partir del cual he desarrollado estas ideas sobre ethos, debe su origen a la estructura discursiva aprendida durante las clases del curso de Ética en la Facultad de Estudios Generales Letras de la PUCP, semestre 2006-2, a cargo del filósofo Fidel Tubino). 
sentimos como familiares, pero que no es identificable de manera inmediata con la ética y aquí vamos a proponer la diferencia. La época de la vida en la que "absorbemos" ese horizonte de valores es fundamentalmente la de la infancia y la pubertad, la de la primera socialización, cuando no somos todavía adultos ni tenemos formado un criterio que diferencie lo bueno y lo conveniente de lo malo e inconveniente. El ethos ingresa en nuestro sistema valorativo como por "ósmosis", lo respiramos sin poder decir no a ninguno de sus elementos ni poder discernir qué es lo relevante y qué lo banal. Y de la misma forma como hay que seguir respirando para poder vivir, adquirimos las creencias que hay en nuestro entorno primario porque necesitamos sentir que el mundo que nos rodea es seguro y tiene un sentido (cosmovisión) en el que podemos creer. $\mathrm{Si}$ respiramos un trato amable en un ambiente flexible, lúdico, con normas suficientes y experiencias de solidaridad responsable de nuestros padres, eso es lo que vamos a adquirir. Si por el contrario respiramos maltrato y brusquedad autoritaria en un ambiente rígido, donde el egoísmo individualista autocomplaciente ha sido entronizado, eso adquiriremos. Más allá de su contenido moral, este ethos en que crecimos va a convertirse en el sustrato de nuestra moral y se va a incorporar pasivamente en forma inconsciente durante la época de la niñez, sin que podamos elegir lo contrario. Este ethos primordial, que acompañará al sujeto a pesar de que luego se transforme en una realidad más compleja, dará lugar a los códigos compartidos de valor o consignas comunes en culturas, instituciones, religiones, asociaciones y grupos a los que pertenecemos, a manera de un consenso inconsciente, de facto, esto es, un acuerdo no dicho ni necesitado de debate. Y por supuesto, mientras convivamos con códigos similares a los nuestros no habrá problema; pero en cuanto nos crucemos con otros códigos distintos, se producirá el conflicto y junto con él la oportunidad de esclarecer su sentido.

Entonces, el proceso de formación de la ética va desde la adquisición del ethos, pasando por la formación de códigos valorativos, hasta llegar a la construcción del punto de vista moral. Este último, forma parte ya de un primer ejercicio adulto de la ética, que consiste en descifrar los elementos inconscientes como sentimientos e intuiciones, traducirlos a conceptos racionales y hacerlos explícitos mediante principios morales. Ahora bien, ¿en qué momento las personas estamos en capacidad de realizar este ejercicio crítico? Cuando dejamos la niñez y nos convertimos en adultos o en sujetos éticos conscientes y capaces de diálogo.

Afirmar sin más, que la ética ya se aprendió en casa es una falacia, si comprendemos que la ética es un ejercicio adulto, un proceso consciente e intersubjetivo, que se construye progresivamente. Si llegamos a la constatación de que el ethos aprendido en casa es negativo, repetirlo sería un acto inmoral por definición y una contradicción insostenible, porque sabiendo que portamos presupuestos de poco significado no podemos asumirlos como significativos: la ética no puede ser al mismo tiempo lo bueno y lo malo. Si por el contrario, llegamos a la conclusión de que es positivo, repetirlo también será inmoral, porque supondrá considerar que como adultos no tenemos ninguna capacidad de intervención en la herencia recibida y su aplicación a nuestra vida será mecánica, infantil y lánguida.

Como ha dicho Fidel Tubino, el ethos hay que entenderlo como locus, en el sentido que permite al sujeto moderno,

"reconocer su pertenencia a una tradición (...) su propia historicidad. Esta conciencia hermenéutica, este saber de las pertenencias, le posibilita al discurso ilustrado (se refiere al proyecto moderno de la Ilustración) el descubrimiento de la alteridad como valiosa y la apertura a otros discursos, a otros ethos, a otras culturas, a otras formas de entender y habitar el mundo"(Turbino, 2004).

Quiere decir que el propio ethos, que nunca abandonaremos, tiene una potencialidad que sigue desarrollándose en nosotros $\mathrm{y}$, en los que junto a nosotros, también lo han adquirido; esa expansión tiene la potencialidad de hacernos trascender y hasta cierto punto desindentificarnos de la identidad que nos había conferido originalmente para hacernos trasmigrar hacia la alteridad de los otros ethos. Hay aquí un proceso que recorrer entre la herencia adquirida en "casa" y la conquista conseguida más allá de ella, en los nuevos espacios adultos de aprendizaje, como la universidad y otros entornos relacionales. En ello, se justifica la necesidad de "la enseñanza" o mejor "el interaprendizaje" de una ética que critica sus orígenes, apreciando y haciendo evidente su universo valorativo, al tiempo que liberándose de los determinismos que la acompañan.

El aprendizaje de la ética, por lo tanto, es un imperativo que consiste en ofrecer espacios de reflexión sistemática y formación del pensamiento ético a los estudiantes, poniéndolos al frente de sus propios aprendizajes adquiridos durante los primeros años de vida, ayudándoles a revisar los presupuestos de la historia personal para que emerjan de ella las creencias inconscientes que pretenden determinar sus acciones conscientes. Además, apunta a facilitar el diseño cooperativo de principios racionales que orienten su acción adulta cotidiana y profesional y justifiquen, al mismo tiempo, el mejor proyecto de vida que quieran escoger en el futuro. Finalmente, mediante el ensayo de situaciones similares, casos y dilemas morales, busca delinear una vida ética activa y consciente, comprometida con las decisiones valiosas posibles que deban tomar, más aún en contextos como el nuestro, definiendo los criterios prácticos que guíen la acción profesional concreta en su compromiso de responsabilidad social (UNALM, 2006).

En ese sentido, los cursos de ética como parte de la propuesta humanística de formación universitaria, no son de ningún modo un adoctrinamiento de manual antiguo ni una simple transmisión de códigos morales a los estudiantes para que puedan ser buenos profesionales.

En el caso de la UNALM, concebimos los cursos de ética con su formalidad académica como una de tantas 
iniciativas que confluyen en la formación ética de nuestros estudiantes, y como parte integral del marco más amplio de la cultura ética donde están inscritos. Dicha cultura de implicancia colectiva, requiere que todos sus actores realicen una concienzuda revisión de los conocimientos, métodos, procedimientos, procesos, proyecciones, acciones y actitudes de los que ya son actualmente responsables, tanto en el escenario mismo del campus como en todos los otros escenarios en que la universidad está presente. Un análisis impostergable para evaluar hasta qué punto nuestro quehacer es coherente con las convicciones y principios que consideramos más valiosos. Esta revisión de las perspectivas efectuada en espacios comunes y en forma colectiva permitirá clarificar los horizontes de valor compartidos y revitalizar la cultura ética ya existente.

El compromiso con la ética nos reportará conciencia, capacidad de manejo de los recursos y capacidad de superación de los condicionamientos para la toma responsable de decisiones. En un primer nivel, este ejercicio mostrará el recorrido que ha seguido la estructuración de la ética a nivel personal para esclarecer de qué manera podemos, a partir de esa toma de conciencia, ser sujetos éticos consistentes. Y en un nivel no posterior sino paralelo, revelará el camino que podría seguirse para reestructurar colectivamente la cultura ética de un grupo.

No se puede ser adulto y vivir con la ética de la infancia; no se puede pretender ser una institución constituida y funcionar de manera fragmentada, sin conciencia la identidad compartida. Cuando se cree haber alcanzado madurez, hay que comprender sistemáticamente cómo se ha formado la ética para poder interpretarla y formular nuevos consensos. Sin embargo, muchas veces lo aprendido en casa o en la institución no ha sido tan saludable, solo que las personas no se atreven a criticarlo, porque lo aprendido es el suelo que se pisa, el que da seguridad y estabilidad, aunque, al mismo tiempo, lo que genera dependencia. Esto último es una razón poderosa para no cuestionarlo. Es menos doloroso aceptar y enorgullecerse de las enseñanzas de los padres que reconocer que a veces enseñaron discriminación, mentira o aprovechamiento de las circunstancias (¿"viveza criolla"? $)^{6}$

\section{Tercera Suposición: "Bueno pues, finalmente la ética ya depende de cada uno".}

Esta última frase a examinarse, parece esconder individualismo, motivo cultural básico de nuestra época, el cual, según Charles Taylor, instrumentaliza las relaciones entre las personas y entre ellas con la naturaleza. Todos somos individualistas, así hemos crecido y comprendido

6Para ampliar esta idea, leer la interpretación de Gonzalo Portocarrero sobre la cultura de la viveza criolla, la cual hunde sus raíces en la historia del Perú colonial y se explica en relación a un cierto goce en la transgresión de la norma, al tiempo que en la supervivencia ante el abuso y las contrariedades socio-culturales, en: PORTOCARRERO, Gonzalo. 2004. Rostros criollos del mal. Cultura y transgresión en la sociedad peruana. Lima: Red para el Desarrollo de las Ciencias Sociales en el Perú. la vida.

"Sin duda, suprimir los viejos órdenes ha ampliado inmensamente el alcance de la razón instrumental. Una vez que la sociedad deja de tener una estructura sagrada, una vez que las convenciones sociales y los modos de actuar dejan de estar asentados en el orden de las cosas o en la voluntad de Dios, están en cierto sentido a disposición de cualquiera. Pueden volver a concebirse con todas sus consecuencias, teniendo la felicidad y el bienestar de los individuos como meta. La norma que se aplica entonces en lo sucesivo es la de la razón instrumental. De forma similar, una vez que las criaturas que nos rodean pierden el significado que correspondía a su lugar en la cadena del ser, están abiertas a que se las trate como materias primas o instrumentos de nuestros proyectos"(Taylor, 1990).

Es parte de esta época. Seguramente las personas vivimos, trabajamos y perseguimos objetivos comunes, pero en el fondo cada cual cree que sus ideas, aspiraciones y valores están por encima de los de los otros. No obstante, creer que la ética depende en última instancia de mí mismo es dar por sentado que mi verdad es la única y que el otro está poco menos que equivocado, siendo entonces inútil esperar algún aporte significativo de su parte. Una perspectiva bastante egocéntrica que impide el intercambio para el descubrimiento de la complementariedad por medio de la experiencia humana del encuentro.

A continuación pongo un par de ejemplos cotidianos que reflejan lo que digo. Dado que en la UNALM, el curso de Ética es un curso de la etapa de los estudios generales, pero hasta hace poco tiempo pero contaba con un número suficiente de docentes para esa labor, era común que desde una o dos semanas antes de iniciar cada semestre vinieran decenas de estudiantes a las oficinas de los profesores, con el interés de solicitar una ampliación de vacante. A continuación se reproduce una escena típica: -Este ... profe, disculpe, por favor, ¿puedo entrar?. Profe, ¿si se acuerda de mí, no? Yo llevé un curso con usted en el primer ciclo. Profe, yo ya estoy al final de la carrera $y$ no he llevado su curso porque nunca he alcanzado vacante... ¿usted cree que podrá ampliarme una para mí?" Entonces, el profesor, le responde, comentándole brevemente los motivos por los que no le es posible ampliar más vacantes y le dice: “¿Conoces a esos tres compañeros tuyos que acaban de salir de mi oficina? Vinieron por la misma razón que tú. Ponte de acuerdo con ellos, organícense y si gustan me llaman para conversar sobre el problema, encontrar un argumento adecuado y plantearlo a las autoridades para que propongan una solución, por ejemplo, contratar más docentes". A lo cual, el alumno dice: - "Ah... mire, pues, ¿puede ser, no? Sí profe... Bueno. Entonces, ¿no tendrá una... sólo una vacante para mí? Ya pues, profe... yo no le voy a decir a nadie que usted me firmó...".

Fíjense bien en esta lógica. El individualismo de pensar en el beneficio que reportará para uno la satisfacción de su necesidad por encima de la necesidad de los otros, es una acción que comienza y termina en sí mismo e 
imposibilita desarrollar vínculos con ellos, borrando del horizonte la dimensión relacional. Es la racionalidad de quienes sólo piensan en conseguir sus propios objetivos y metas, porque no son capaces de comprenderse como miembros de la misma comunidad junto a otras personas y por eso se saben aislados de sus intereses, su historia $\mathrm{y}$ sus proyectos, que finalmente, son comunes a todos. ¿Acaso la imposibilidad de construir "un nosotros diverso", como dijera Carlos Iván Degregori, en medio de un Perú tan diverso y fragmentado, no está relacionada con la incapacidad para vincularse con los otros, cargando y superando los peruanos, su historia compleja? Estos horizontes en los que están inscritos los han influido desde siempre y son los entornos cargados de cuestiones valiosas en medio de los cuales han desarrollado, horizontes que están en ellos y que no podemos eludir:

"Las cosas adquieren importancia contra un fondo de inteligibilidad. Llamaremos a esto horizonte. Se deduce que una de las cosas que no podemos hacer, si tenemos que definirnos significativamente, es suprimir o negar los horizontes contra los que las cosas adquieren significación para nosotros. Este es el tipo de paso contraproducente que se da con frecuencia en nuestra civilización subjetivista. Al acentuar la legitimidad de la elección entre ciertas opciones, muy a menudo nos encontramos con que privamos a las opciones de su significación"7

Volviendo al ejemplo de los estudiantes. No se pretende afirmar que en estas actitudes haya maldad por parte de ellos, pero en todo caso, se espera que los docentes, como adultos que son y que tienen el encargo y la responsabilidad de formar a los más jóvenes, asuman la responsabilidad de mostrarles cuánto se pierde con el individualismo, cuánto se debilita el tejido social con la ausencia del debate público en torno a los intereses comunes, cómo es que las personas han llegado a ser presa fácil entre las manos de quienes tienen el poder que ellas mismas les han conferido y que las instrumentalizan porque han hipotecado su libertad política.

“(...) una nueva forma específicamente moderna de despotismo, a la que Tocqueville llama despotismo "blando". No será una tiranía de terror y opresión como las de tiempos pretéritos. El gobierno será suave y paternalista. Puede que mantenga incluso formas democráticas, con elecciones periódicas. Pero en realidad, todo se regirá por un "inmenso poder tutelar" sobre el que la gente tendrá poco control. La única defensa contra ello, piensa Tocqueville, consiste en una vigorosa cultura política en la que se valore la participación, tanto en los diversos niveles de gobierno como en asociaciones voluntarias. Pero el atomismo del individuo absorto en sí mismo milita en contra de esto. Cuando disminuye la participación, cuando se extinguen las asociaciones laterales que operaban como vehículo de la misma, el ciudadano individual se queda solo frente al vasto Estado burocrático y se siente, con razón, impotente. Con ello se desmotiva al ciudadano aún más, y se cierra el círculo vicioso del despotismo blando". 8

Luego, decir que la ética ya depende de cada uno es una afirmación peligrosamente individualista. Remite a figuras como Hitler, Bush o Fujimori, que se obstinaron en el autoengaño de creer que sus criterios eran los mejores y tomaron las decisiones más erradas con desastrosas consecuencias para la historia y la sociedad. Claro que no, la ética no depende de cada uno ni se lleva dentro sin más, sino que debería ir apareciendo como resultado de una práctica deliberativa en una comunidad ética que es inclusiva y dialogante, tal como piensa Jurgen Habermas y que ejercita la oportunidad para encontrar el mejor argumento capaz de sustentar los ideales, valores y proyectos comunes. Una comunidad que no excluye a ninguna voz y que no impone sus argumentos a nadie, sino que los construye en competencia cooperativa con todos.

"Puesto que dichos procesos de entendimiento y forma de vida tienen ciertos aspectos estructurales comunes. Las teorías que se hallan en la tradición de Hegel, Humboldt y G. H. Mead han admitido estos indicios para mostrar que las acciones comunicativas se entretejen con suposiciones recíprocas, igual que las formas de vida comunicativas lo están con las relaciones de reconocimiento recíproco, y en esa medida tienen un contenido normativo. De estos análisis se deduce que la moral se refiere, por la forma y por la estructura de las perspectivas de la socialización intersubjetivamente no deformada, a un sentido genuino, independiente del bien individual"(Habermas, 2000).

Otro caso es el de las condiciones o lineamientos para la investigación. Desde hace ya varias décadas la investigación en el ámbito académico se concibe en perspectiva interdisciplinaria y colectiva. También en el caso de la Universidad Agraria, algunas iniciativas surgidas en ella han ido sembrando cuestiones de gran significación en el campo de la enseñanza-aprendizajeinvestigación. Sin embargo, en sentido práctico dichas normas no reflejan estas prácticas valiosas ni sus propuestas. Por ejemplo, diferencian claramente, "investigadores líderes" de "colaboradores". Estas denominaciones no son gratuitas. El lenguaje refleja el ethos de una institución y habría que preguntarse si esto pasa con dichos conceptos, o sea, si reflejan las consideraciones y convicciones y si no se debería acaso modificarlos. En tal sentido, llamar "líder" al coordinador de un proyecto podría prestarse a confusión, dejando entender que este sería un jefe que manda, antes que un coordinador-motivador de las líneas de investigación. Los investigadores tienen diversidad de capacidades y experiencia, pero eso no significa que haya un líder más capacitado que los otros miembros o el más importante a quien se deba seguir. Este coordinador es uno de los colaboradores cuya función es la de motivar, integrar y sintetizar, a razón de que posee alguna trayectoria o mayor experiencia, respecto al contenido del proyecto de investigación. De hecho, las normas para investigar y publicar también obligan a indicar con claridad el grado ${ }^{8 i}$ dem, pp. 44-45. 
de intervención del líder y sus colaboradores asignando porcentajes a la participación de cada uno, resultando como consecuencia que los que colocan un número más alto reciben mayor puntaje y los que colocan uno más bajo, menor puntaje. Eso hace recordar a un clásico profesor de colegio primario de otros tiempos que, en vez de promover el trabajo colaborativo para el aprendizaje, hacía dos cosas: por un lado, incitaba a la competencia individualista, repartiendo puntos a los niños más aplicados, afanosos y competitivos que podían hasta enemistarse con sus compañeritos de carpeta con tal de alcanzar un punto más en la calificación y distinguirse de esta manera como "los mejores"; y por otro, desatendía las diversas formas de aprendizaje o los distintos condicionamientos y saberes previos que traían los otros niños, desde su perspectiva estrecha, "los peores".

En última instancia, parece no existir un convencimiento acerca de los beneficios de las investigaciones colectivas para la formación de la comunidad de aprendizaje, parece que se sugiere repartir la torta en partes desiguales sin considerar el carácter cualitativo de las investigaciones. Todo esto refleja una lógica jerárquica y que, además, no está interesada en coordinar los mecanismos de socialización para el debate público de los resultados y hallazgos en el espacio de la comunidad académica.

Así parece ser. Se vive en una cultura individualista que ha truncado sus condiciones para asociarse y organizarse, para proyectarse al futuro en planes colectivos de acción y tender redes sociales con miras a un mayor impacto de cara a la comunidad y la sociedad. Parecería que el mayor logro asociativo fuera el facebook o el twiter, donde de los 3500 "amigos" de los contactos personales, ninguno es verdaderamente un amigo ni mucho menos un socio. ¿Será que la virtualidad de las redes sociales, siendo excelentes herramientas de comunicación e intercambio, refleja en este caso la incapacidad de relacionalidad entre las personas de esta época y entre ellas y el medio ambiente desprotegido del impacto agresivo?

Para terminar, quisiera invitarlos a compartir estas ideas con otros y así derivar nuevas conclusiones, a partir del examen de otras frases cotidianas que también se emplean, no tomándolas como una catástrofe sino como un reto para avanzar en la renovación de los contenidos y significados valiosos de la "cultura ética institucional".

\section{Conclusiones}

La ética tiene un potencial humanizador en el proceso de formación de los estudiantes universitarios y su tarea debe incluir el esclarecimiento de los presupuestos científico-técnicos con una orientación moral de cara al bien. Esta debe ser una ética crítica con las prácticas, procedimientos, métodos e instrumentos que utiliza la ciencia para manejar el entorno, sin suponer que puede conducirse sola como si fuera un dispositivo inteligente que no requiere de la intervención y cuidado humanos. Esa es la difícil tarea de la ética: trascender algunas suposiciones referidas a la idealización de los primeros elementos valiosos adquiridos en el hogar y el entorno primario, como la suposición de que todo lo adquirido es bueno. Para ello, necesita pasar el ethos primero por el tamiz de una racionalidad dialógica que actúa como filtro crítico en el debate colectivo con los otros encargados de la formación y seguir construyendo con ellos, así como perfeccionando, los nuevos sentidos compartidos de una racionalidad y una praxis moral coherentes. La formación de una conciencia crítica entre los estudiantes pasa por el compromiso colectivo de la universidad con un proyecto de ética capaz de impulsar la superación de los fines individuales y el fortalecimiento de las redes sociales en la praxis profesional, una ética capaz de orientar la ciencia y sus procedimientos hacia la consecución de objetivos comunes, no sólo los que se persiguen como institución sino los que están más allá de ella, una ética que represente el ejercicio de tejer relaciones cada vez más resistentes y armoniosas de justicia, inclusión y desarrollo, con miras a una convivencia más humana y digna para todos.

\section{Literatura citada}

Bunge, M. 2016. La Ciencia. Su método y su filosofía. Madrid: Laetoli. Madrid: Laetoli. p. 6.

Freud, S. [1900] 1988. La interpretación de los sueños. En "Obras completas" 3, XVIII. Buenos Aires: Hispamérica Ediciones.

Gadamer, Hans-George. 1981. Acerca de la disposición natural de los hombres para la filosofía. En "La razón en la época de la ciencia". Barcelona: Editorial Alfa Argentina. p. 87.

Habermas, J. 2000. Ética discursiva. En Carlos Gómez "Doce textos fundamentales de la Ética del siglo XX". Madrid: Alianza Editorial.

Taylor, Charles Ética de la autenticidad. Barcelona: Herder. pp. 175-176.

Tubino Arias-Schereiber, F. 2004. Ciudadanías complejas y diversidad cultural. En "Ciudadanías inconclusas”. Lima: PUCP Fondo Editorial. , p. 172.

UNALM, 2006. Dpto. Ciencias Humanas Perfil del Departamento de Ciencias Humanas. Lima: Departamento de Ciencias Humanas, UNALM. 